\title{
Accurate Location of Transformer Hottest Spot by FEM and Thermal Models
}

\author{
Dr. V. Rajini \\ SSN College of Engg \\ India
}

\begin{abstract}
The formation of hotspots in the power transformers is one of the major threats for the life of the transformer. Therefore, the hot spot temperature value is an important parameter governing the life expectancy of a power transformer. This paper presents an approach to estimate and locate the hotspot accurately by considering the losses distributed across the transformer geometry. The distributed equivalent electrical circuit based on the thermal electrical analogy, is developed for a $50 \mathrm{kVA}$ transformer. The results are also compared with the classical approach of estimating the hot spot temperature.
\end{abstract}

\section{Keywords}

Distributed losses, FEM, Hotspots, Thermal electrical analogy, Temperature profile, Thermal model.

\section{INTRODUCTION}

Power transformers are the most vital and costly investment in a power system. The life of a power transformer is mostly governed by its hot-spot temperature. The winding hot spot temperature is the main factor limiting the loadability of a power transformer. Higher winding hot spot temperature causes degradation of the insulating materials and results in the formation of gas bubbles which facilitates the deterioration of transformer oil. The insulating oil changes its chemical properties and causes dissociation of oil, increased pressure in the tank because of the gases formed during the supposed chemical reactions which enhances the chances of tank explosion and fire hazards. The change in the electric and magnetic properties of the core and coil again result in increased losses and increased heat generation and accelerate the above discussed effects. Among these consequences, insulation deterioration is economically important. Insulation being very costly, its deterioration is undesirable as it is the main factor of transformer aging.

With temperature and time, the cellulose insulation undergoes a depolymerization process. As the cellulose chain gets shorter, the mechanical property of paper such as tensile strength and elasticity degrades. Eventually the paper become brittle and is not capable of withstanding short circuit forces and even normal vibrations that are part of transformer life. This situation characterizes the end of life of the solid insulation. Since it is not reversible, it also defines the transformer end of life. Hence, sustained efforts have been made to monitor the hot spot temperature to take advantage of cool ambient temperature, extend the transformer life while providing emergency overloading capabilities and taking advantage of market opportunities.

For several decades IEEE and IEC loading guides $[1,2]$ have been providing guidelines for the calculation of the winding hottest spot temperature from data that can be conveniently measured and parameters derived from temperature rise test or manufacturer calculations. The basic calculation method relies on the measurement of oil temperature at the top of the transformer tank (top-oil temperature) and a calculation of the temperature difference between the winding hottest spot and the top oil. This temperature rise is provided by the manufacturer, based on his modeling of oil flow and losses distribution in the winding. Thereafter the hot-spot temperature can be computed for any load using standard relations.

It is also recognized that a sudden increase in load current may cause an unexpected high peak in the winding hot-spot temperature and hence the above method of estimation is inadequate. Also, these approaches do not take in to account, the winding eddy losses and stray loss in other structured parts of transformer. As a result, the direct measurement of winding temperature using fiber optic probes is always found to be greater than the estimated values. Attempts have been made by several researchers to estimate the hot spot temperature accurately $[3,4,5$ and 6]. The stray loss evaluation is an essential aspect to calculate hot spot temperature. The stray losses in transformer is caused by the time variable leakage flux which induces emf \& circulates eddy currents in the winding conductors and in the other conducting parts of transformer like tank wall, core, clamps etc[7,8,9]. Evaluation of stray losses can be done more accurately by FEM. Hot spot temperature estimation for a dry type transformer[6] and for a ONAN power transformer[10], is also done in by a similar approach by thermal electrical analogy explained in IEC loading guides. This paper presents a new approach to calculate the hot spot temperature taking in to account the losses distributed across the transformer geometry. The distributed thermal model developed in this paper can be used to locate the hot spot accurately.

\section{PROBLEM GEOMETRY}

This paper considers a $50 \mathrm{kVA}, 20.5 / 0.41 \mathrm{kV}$, ONAN unit. The LV(Low Voltage) winding consists of $4.5 \times 6.5 \mathrm{~mm}$ flat conductors. The high voltage winding consists of round conductors of $0.6 \mathrm{~mm}$ diameter with 19 layers of 142 conductors in each. The two dimensional cross section of the transformer essential parts is shown in figure 1. The transformer modeled is as shown in Fig 2.The LV winding has been modeled in 3 layers with 54 conductors. The windings are sandwiched between layers of oil impregnated paper insulation. The windings are shown in Fig 3. The properties of materials used for FEM analysis of transformer are tabulated in Table 1. The tank shunt is neglected in the analysis. The half symmetry model is solved using FEM. After intense mesh analysis, the field solution is obtained as shown below in the Fig 4. The magneto static solution in FEM is used to calculate losses in various sections of the transformer model. Here, the winding eddy losses are neglected. 


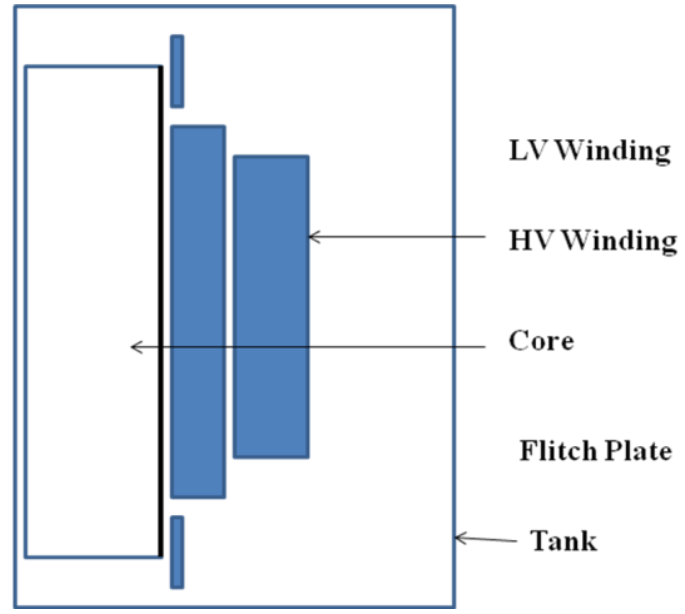

Fig 1 Two dimensional transformer cross section showing essential parts

\section{DEVELOPMENT OF THERMAL MODEL \\ 3.1 The Classical Model}

The thermal model of the transformer is formed from the equivalent electrical circuit simulating the thermal behavior of each element. Electrical equivalents are built for each element and they are used to develop the thermal model using thermal electrical analogy $[2,3]$

A thermal process is defined by energy balance equation

where,

$$
q=C_{t h} \times \frac{d \theta}{d t}+\frac{\theta-\theta_{a m b}}{R_{t h}}
$$

$\mathrm{q}$ is the heat generation,

Cth is the thermal capacitance,

$\theta$ is temperature,

$\mathrm{R}_{\mathrm{th}}$ is the thermal resistance,

$\theta_{\mathrm{amb}}$ is the ambient temperature.

A simple electrical RC circuit yields a similar equation based on Kirchoff's law,

$$
q=C_{e l} \times \frac{d u}{d t}+\frac{u}{R_{e l}}
$$

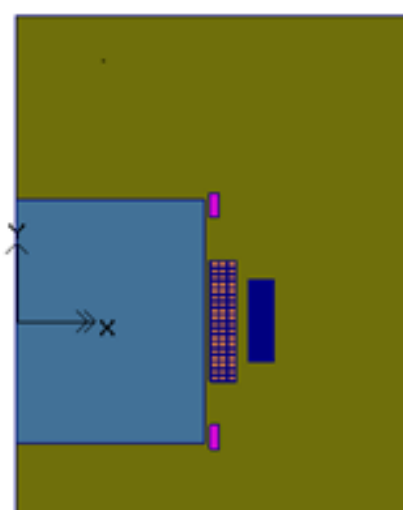

Fig 2. Model of transformer in FEM

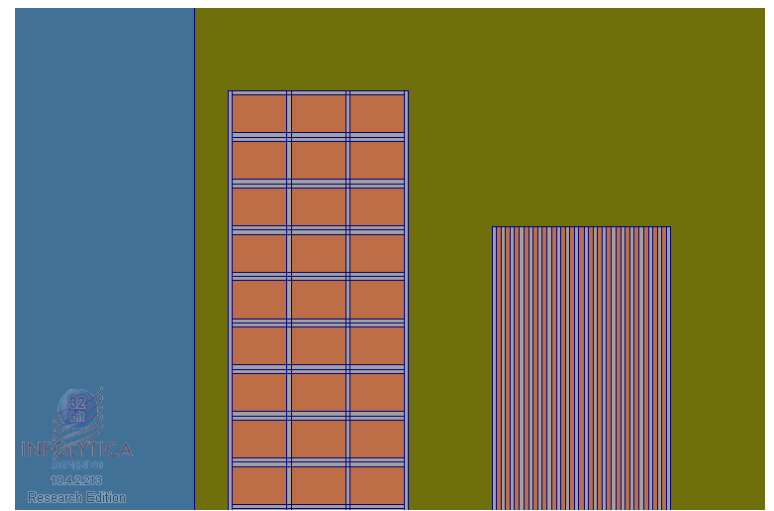

Fig 3. Transformer model showing the core and windings

where

$i$ is the electrical current,

$\mathrm{C}_{\mathrm{el}}$ is the electrical capacitance,

$\mathrm{R}_{\mathrm{el}}$ is the electrical resistance,

$\mathrm{u}$ is the electrical voltage

Comparing equations (1) and (2) we obtain the analogy between electrical and thermal processes. Table 2 describes the thermal- electrical analogy and Fig 5. gives the electrical equivalent used for simulating the thermal behavior.

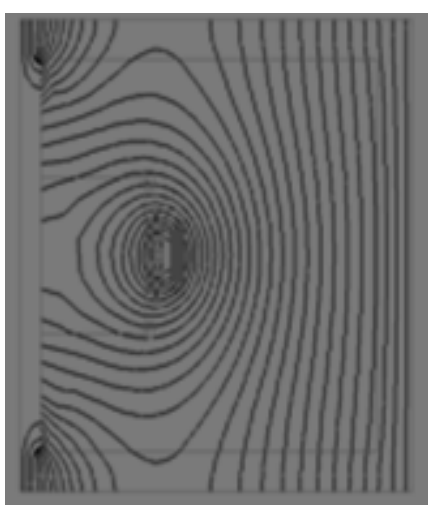

Fig 4. Field pattern in transformer

Table 1 Properties of Materials Used In FEM

\begin{tabular}{|l|l|l|l|l|}
\hline Properties & $\begin{array}{l}\text { Ai } \\
\text { r }\end{array}$ & CRS & OIP & $\begin{array}{l}\text { Coppe } \\
\text { r }\end{array}$ \\
\hline Permeability & 1 & - & 0.8 & 1 \\
\hline Conductivity & 0 & 0 & - & - \\
\hline Electric Permittivity & 1 & 1 & 3.1 & 1 \\
\hline Thermal Conductivity & $\begin{array}{l}0.0 \\
2\end{array}$ & 49.8 & 0.26 & 386 \\
\hline Thermal Heat Capacity & $\begin{array}{l}1.0 \\
0\end{array}$ & 448 & 0.6 & 383.1 \\
\hline Mass Density & 1.2 & 7600 & 1.2 & 8954 \\
\hline
\end{tabular}


Table 2.Thermal-Electrical Analogy

\begin{tabular}{|c|l|}
\hline Thermal & Electrical \\
\hline Heat generated $\mathrm{q}$ & Current $\mathrm{i}$ \\
\hline Temperature $\theta$ & Voltage $\mathrm{u}$ \\
\hline Resistance Rth & Resistance Rel \\
\hline Capacitance Cth & Capacitance Cel \\
\hline
\end{tabular}

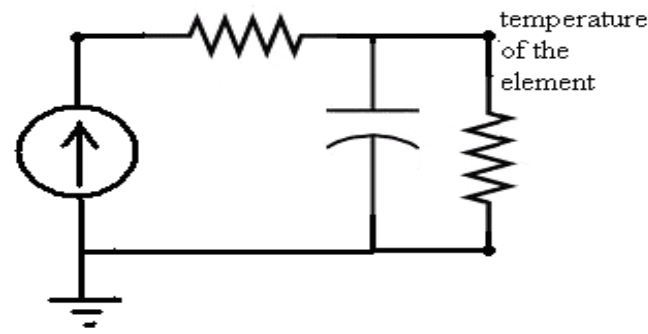

Fig 5. Equivalent circuit to simulate the thermal behavior

In order to develop the thermal model the following points are to be noted:

i) Oil is a dissipating medium and it does not generate heat, so no losses are produced. Hence there are no sources in case of oil elements.

ii) The heat dissipation from tank to ambient is represented by means of thermal resistances connecting the outer nodes of tank to ambient.

iii) The ambient is taken as ground in the circuit model. It is at a potential equal to $20^{\circ} \mathrm{C}$. This says that the thermal model yields temperature rise above ambient temperature.

iv) Symmetry is observed

The sources in the model represent the heat generated by each element due to the losses. The loss in the winding, copper loss given by $I^{2} R$ is calculated using the value of current and resistance in each section. The loss in the core is calculated using the formulae

$$
P=K_{h} f B^{1.6}+K_{e}(s f B)^{2}
$$

is the core loss

$\mathrm{K}_{\mathrm{e}}$ and $\mathrm{K}_{\mathrm{h}}$ are material constants

$\mathrm{f}$ is the frequency of the alternating flux,

$\mathrm{B}$ is the maximum value of operating flux density,

$\mathrm{s}$ is the space factor

The heat dissipation by elements is represented by connecting resistors horizontally and vertically.Capacitors represent the storage of heat. The values of resistances and capacitances are calculated using the following formulae

$$
\mathrm{R}_{\mathrm{th}}=\frac{\Delta \theta_{\text {oil-rated }}}{\mathrm{q}}
$$

where,

$\mathrm{R}_{\mathrm{th}}$ is the thermal resistance,

$\Delta \theta_{\text {oil-rated }}$ is the rated top oil temperature rise, $\mathrm{q}$ is the heat generated.

$$
\mathrm{C}_{\mathrm{th}}=\text { mass } \times \text { specific heat capacity }
$$

$$
\theta_{\mathrm{H}}=\theta_{\mathrm{A}}+\Delta \theta_{\mathrm{TO}}+\Delta \theta_{\mathrm{H}}
$$

where

$\theta_{\mathrm{A}}$ is the ambient temperature, ${ }^{\circ} \mathrm{C}$.

$\Delta \theta_{\mathrm{TO}}$ is the oil temperature rise over ambient, ${ }^{\circ} \mathrm{C}$. $\Delta \theta_{\mathrm{H}}$ is the hot spot temperature above top oil ${ }^{\circ} \mathrm{C}$. $\theta_{\mathrm{H}}$ is the ultimate hot spot temperature, ${ }^{\circ} \mathrm{C}$.

Considering these assumptions, thermal model is developed for top oil, winding hotspot and bottom oil. These models are simulated to find the output voltage which is analogous to the temperature of these areas. These models are cascaded to obtain the overall temperature value. Fig 6 . shows the thermal model for top oil, winding hotspot and bottom oil respectively. These models are cascaded to obtain the overall temperature value. The temperature rise is found to be $114.9^{\circ} \mathrm{C}$ above the ambient

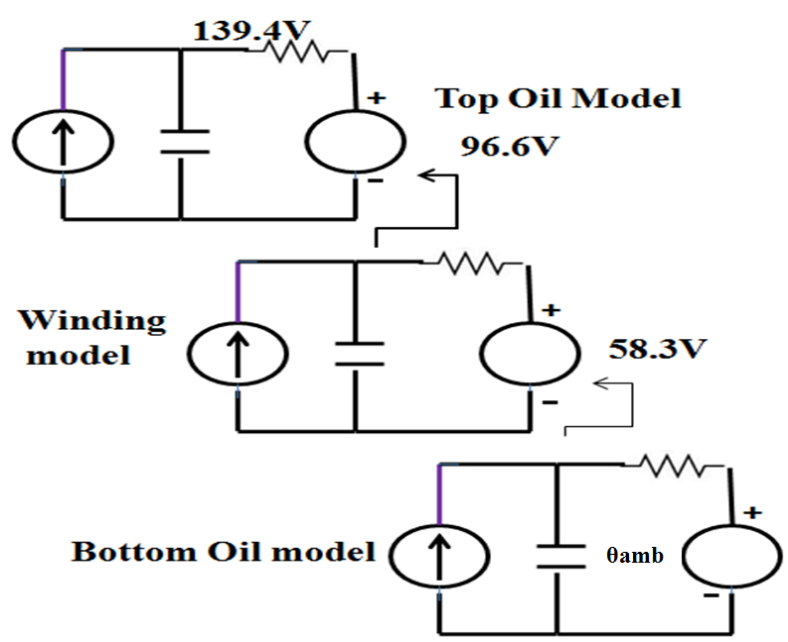

Fig 6. Lumped model for temperature rise

\subsection{Mathematical Model - IEC}

The hot-spot temperature can also be computed for any load using the standard relations given by IEC Loading guide .The top oil rise is calculated as a first order exponential response from the initial temperature state to the final temperature state.

$$
\begin{gathered}
\Delta \theta_{\mathrm{TO}}=\left[\Delta \theta_{\mathrm{TO}, \mathrm{U}}-\Delta \theta_{\mathrm{TO}, \mathrm{i}}\right]\left[1-\mathrm{e}^{-\frac{\mathrm{t}}{\tau_{0}}}\right]+\Delta \theta_{\mathrm{TO}, \mathrm{i}}(7) \\
\tau_{\mathrm{TO}} \frac{\mathrm{d} \Delta \theta_{\mathrm{TO}}}{\mathrm{dt}}=\left[\Delta \theta_{\mathrm{TO}, \mathrm{U}}-\Delta \theta_{\mathrm{TO}}\right] \\
\Delta \theta_{\mathrm{TO}, \mathrm{U}}=\Delta \theta_{\mathrm{TO}-\mathrm{R}}\left[\frac{1+\mathrm{RK}^{2}}{1+\mathrm{R}}\right]^{\mathrm{n}} \\
\theta_{H S}=\theta_{T O}+\Delta \theta_{H R}\left(\frac{I}{I_{R}}\right)^{2 m}
\end{gathered}
$$

where, 
$\Delta \theta_{\mathrm{TO}}$ is the top oil temperature rise over ambient temperature $\Delta \theta_{\mathrm{TO}, \mathrm{U}}$ is the ultimate top oil temperature rise over ambient temperature

$\mathrm{K}$ is the load current per unit

$\tau_{\mathrm{TO}}$ is the top oil rise time constant

$\Delta \theta_{\mathrm{TO}-\mathrm{R}}$ is the rated top oil temperature rise over ambient temperature

$\mathrm{R}$ is the ratio of load losses at rated load to no load losses

$\mathrm{n}$ is an empirically derived exponent that depends on the cooling method

I = Load current

$\mathrm{I}_{\mathrm{R}}=$ Rated current

$\mathrm{m}=$ Winding exponent

The hot spot temperature is calculated at each instant of the transformer operation at different loading conditions and variable ambient temperature in this paper. The mathematical model is developedtions using the heat conduction equations that govern the dynamics of heat flow. The model is solved using MATLAB. The load cycle used for the simulation model is as per IEC recommend The temperature rise obtained from this model is found to be $116.7^{\circ} \mathrm{C}$.

\section{IMPROVED DISTRIBUTED THERMAL MODEL}

The lumped parameter model yields the hottest temperature but, does not provide the exact location of it. In an attempt to find the accurate location of hotspot in the transformer geometry, the various losses distributed across the geometry must be considered. Hence, the transformer geometry is divided in to various segments and the electrical analogies are obtained for each of the segment and are connected in a manner similar to the connection in the actual geometry. This provides the temperature profile across geometry using which, the accurate locations of hotspots are found. The transformer geometry is divided into segments for convenience. The thermal resistance exhibited by a segment is equal to the temperature drop across the segment divided by the total amount of heat the section has to dissipate and this value has to be equal to the total heat generated by all the elements geometrically enclosed within the segment. Fig 7. shows the division of segments along the transformer geometry. The segmentation is such that the segment S1 includes the transformer core, oil duct and tank, where the heat generated by the core is dissipated through its surrounding elements. Similarly, segments S2 and S3 are divided likewise, where heat is generated by the LV and the HV respectively. Each segment is again divided into number of elements. Figure 8 shows the division of segment $\mathrm{S} 1$ in to number of elements and the electrical equivalent of thermal model is obtained for each of this element in a manner discussed above. Division of S2 and S3 are not shown here but are done similar to that of $\mathrm{S} 1$. The overall resistance of the segments formed is calculated. This resistance is distributed across the elements in that segment.

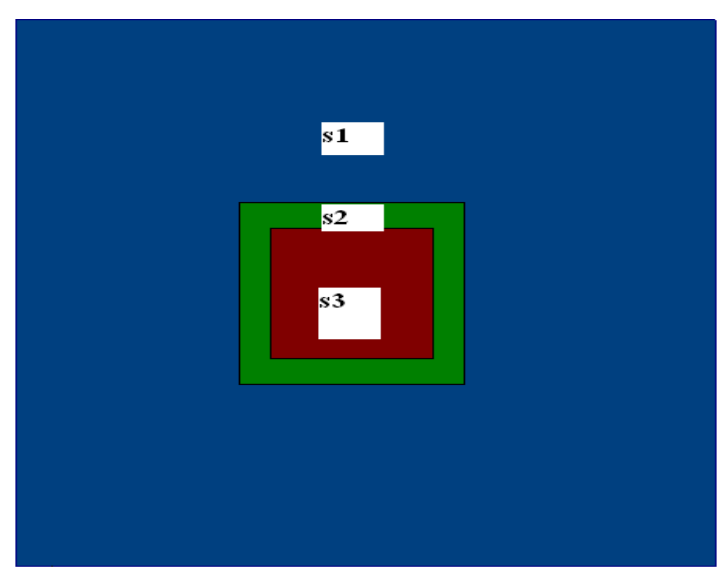

Fig7. Segmentation of transformer geometry

The calculated resistance multiplied by the total surface area of dissipation will be proportional to the specific resistance exhibited by that section of elements. Depending upon the surface area of individual element, this value is used to calculate the resistance exhibited by individual element. The capacitance is calculated as explained before. With a motive to locate hotspots accurately, the distributed thermal model of entire transformer geometry is solved to get the output distribution of all nodes which directly gives the temperature profile of the transformer and hence, the location of the hotspot. The hottest temperature obtained by this method is compared with the values obtained by the classical approach discussed in the previous section.

\section{RESULTS AND COMPARISON}

From the distributed thermal model, it is found that hotspots are at right hand side end of the HV winding area. The hottest temperature is found to be $205.5^{\circ} \mathrm{CV}$. The other high temperatures are $197.9,201,203.6$, and $204^{\circ} \mathrm{C}$. Fig 10 . shows 


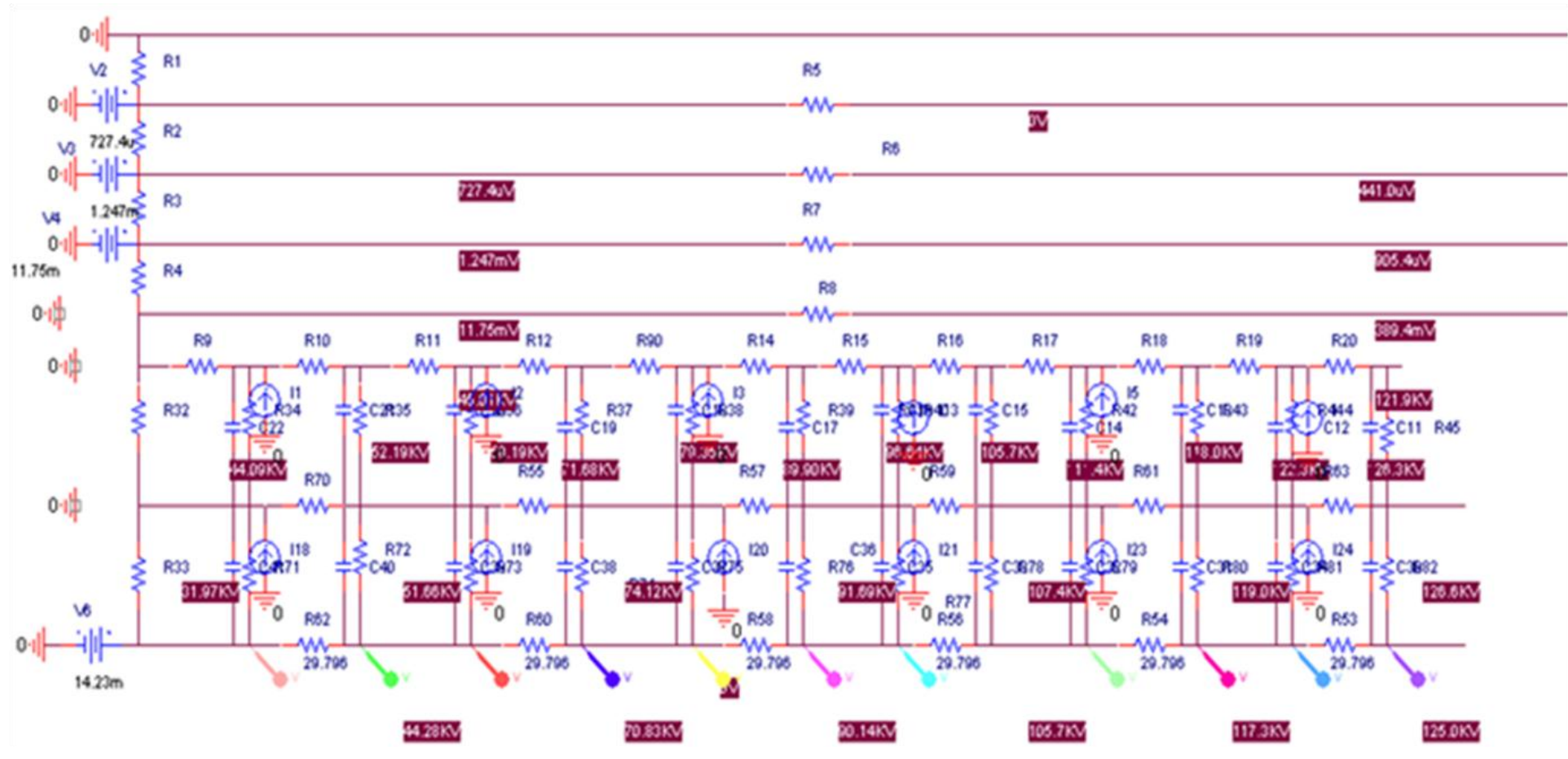

Fig 8. Electrical Analogy for a section of transformer

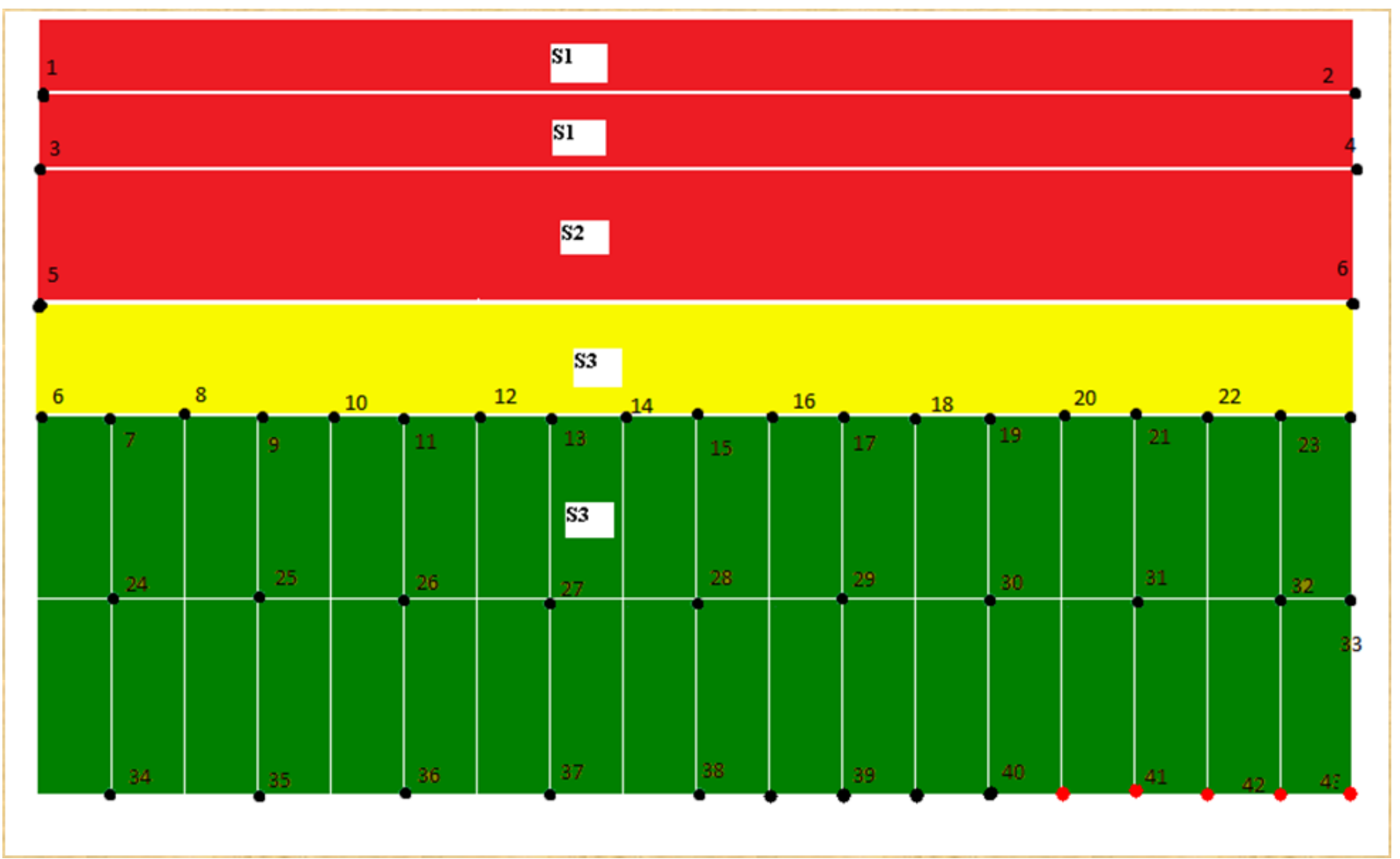

Fig 9. Section showing the Exact location of Hotspots 


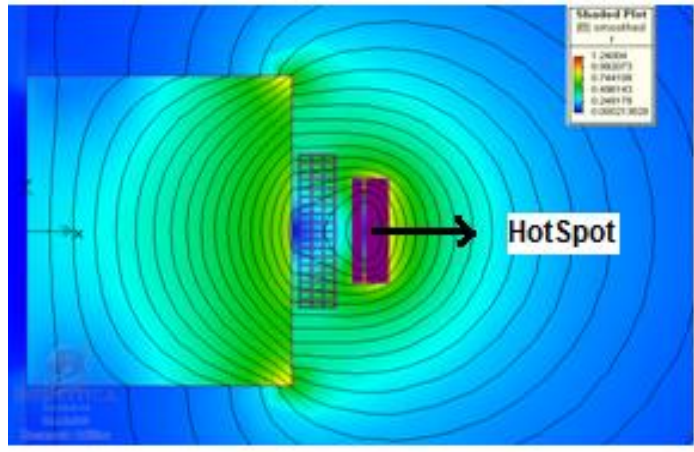

Fig 10. Approximate hotspot location as obtained in ANSYS

Hot spots located on the right side of HV winding and they are indicated in red. Only those sections in which the temperature profile is high are shown in figure 10 and figure11. The temperature profile across the transformer geometry is obtained using ANSYS also for comparison The approximate location of hotspot as obtained from ANSYS is given in figure 12.It shows that the hotspot is located near HV coil. The hotspot is found to be $114.9^{\circ} \mathrm{C}$ above the ambient by the classical approach whereas it is $116.79^{\circ} \mathrm{C}$ by the dynamic mathematical modeling.

\section{CONCLUSIONS}

Presented model provides information about important thermal data for prognosis, simulation and analysis of the transformer operation. A technique to develop a distributed thermal model of power transformer for accurate estimation and location of hottest spot is presented. The core losses of the transformer are computed by determining the flux density. The thermal model is developed for the transformer using thermal electrical analogy. The hotspot temperature obtained from the lumped model is $114.4^{\circ} \mathrm{C}$ whereas it is $116.7^{\circ} \mathrm{C}$ when calculated using the mathematical model developed using simulink (IEC model) and the results agree with each other in good accuracy. But for accurate estimation of temperature and for the location of hottest spot, the new distributed model discussed in this paper is suggested. For the given transformer it is estimated to be $205.5^{\circ} \mathrm{C}$ above ambient and is found to be higher than that obtained from the classical methods. The distributed thermal model considering the losses in the respective part of the transformer geometry also gives the temperature profile from which the other hotspot can be located. This improved model also reaffirms the location of the hottest spot at the rightmost side of the HV winding as predicted using ANSYS. With this new model, it is now possible to locate the hotspot accurately and helps in the accurate placement of sensors for continuous monitoring of such critical spots in real tome service. This methodology can be extended further to develop the thermal model incorporating the stray loss and eddy current loss in the windings also.

\section{REFERENCES}

[1] IEEE C57.91-1995 "IEEE Guide for Loading MineralOil-Immersed Transformers"

[2] IEEE C57.145 "Guide for the Definition of Thermal Duplicate Liquid-Immersed Distribution, Power and Regulating Transformer"

[3] Dejan Susa, Matti Lehtonen, and Hasse Nordman, "Dynamic Thermal Modelling of Power Transformers", IEEE Transactions on Power Delivery, Vol. 20, No. 1, January 2005.

[4] Haritha V V S S, T R Rao, Amit Jain, M Ramamoorty, "Thermal Modeling of Electrical UtilityTransformer Using Finite Element Modeling Technique and ThermalElectrical Analogy", Presented in Third International Conference on Power Systems, Kharagpur, India , December 27-29, 2009.

[5] Ires Iskender, Ali Mamizadeh, " Non Linear Thermal Modeling of Indoor and Outdoor Oil-Immersed Power Transformers",Journal of Electrical Engineering, Vol.60, N0.6, pp 321-327, 2009.

[6] M Ghareh, L Sepahi, " Thermal Modeling of DryTransformers and Estimating Temperature Rise", World Academy of Science, Engineering and Technology, Vol. 45, pp 290- 292, 2008.

[7] Traian Chiulan, Brandusa Pantelimon, “ Theoretical Study on a Thermal Model for Large Power Transformer Units", International Journal of Engineering and Applied Sciences, Vol.4 ,No.4, pp 203- 206, 2008.

[8] G. Swifft, om.S.Molinski, W.Lehn," Fundamental approach to Transformer Modelling - Part I" Theory and equivalent Circuit, IEEE transactions on Power Delivery, Vol.16, No.2, april 2001

[9] Canadian Electrical Association Report 355T822, "Application of Fibre Optic Temperature Sensors to Establishing Transformer Overload Capabilities.," 53 pages, March 1995.

[10] Ankireddypalli S. Reddy, M. Vijaykumar," Hottest Spot and Life Evaluation of Power Transformer using Finite Element", Journal of Theoretical and Applied Information Technology,"pp-238-243, 2005

[11] .Zoran Radokovic,Kurt Feser,"A new method for the calculation of the hotspot temperature in power transformers with ONAN cooling", IEEE Trans on Power Delivery, Vol.18,No.4,pp1284-1292, 2003 